\title{
基于案例分析与翻转课堂的分析化学实验混合式教学实践
}

毛全兴 ${ }^{*}$, 张渝阳, 娄振宁, 王月娇, 许旭, 刘琳

辽宁大学化学院, 沈阳 110036

摘要: 设计并实践了新的分析化学实验教学方法和考评方法。将案例分析与翻转课堂相结合, 进行线上线下混合式 教学。课前教师选择案例并设计问题, 学生分组预习; 课堂上学生讨论分析为主, 教师查漏补缺为辅; 课后学生撰 写报告上传至线上平台, 教师批阅并反馈问题。新的考评方法中, 突出对学生表达能力、逻辑思维能力、团队协作 能力、实验操作水平、实验台与实验室整洁水平的考查评价。实践表明, 新的教学方法有助于提升学生的表达能力 和实验操作水平, 激发学生学习的积极性和挑战高难度学习任务的热情。新的考评方法为评价学生能力提供了可执 行的标准。

关键词: 分析化学实验; 案例分析; 翻转课堂; 混合式教学; 考评方法

中图分类号: G64; O6

\section{Practice of Blended Teaching Mode Based on Case Analysis and Flipped Classroom in Analytical Chemistry Laboratory Teaching}

\author{
Quanxing Mao *, Yuyang Zhang, Zhenning Lou, Yuejiao Wang, Xu Xu, Lin Liu \\ College of Chemistry, Liaoning University, Shenyang 110036, China.
}

\begin{abstract}
We designed and practiced a new teaching and evaluation method in analytical chemistry laboratory teaching. By combing case analysis with flipped classroom, the courses were finished via mixed avenue of online and offline. Before class, teachers selected cases and raised series of questions, while students studied related contents in-teams. In class, students discussed the cases and questions, and teachers checked and made up for deficiencies. After class, students wrote and uploaded their reports, and teachers corrected errors in the reports. The new method evaluated the expressiveness, logical thinking, teamwork, experimental operation and cleanliness of bench and the lab. The practice of the new teaching method helps to improve the performance of students in expressiveness and lab skills, as well as to inspire the passion for leaning and taking challenging tasks. The evaluation method provided feasible criteria for evaluating the ability of students.
\end{abstract}

Key Words: Analytical chemistry laboratory; Case analysis; Flipped class; Blended teaching;

Evaluation method

分析化学实验是化学类专业的主干课程, 也是药学、生命科学、生物工程、环境工程等专业的 重要基础课程。分析化学实验课程的教学目标是通过实验巩固理论知识, 加深对分析化学基本原理 的理解与掌握; 通过对基本实验方法与实验技能的练习, 提升学生的动手、观察、思维、表达等各 方面的能力; 并由此培养学生实事求是的科学态度和学以致用的学习精神。

收稿: 2021-11-01; 录用: 2021-11-15; 网络发表: 2021-12-14

“通讯作者, Email: maoquanxing@1nu.edu.cn

基金资助: 辽宁大学本科教学改革项目(JG2020YBXM019, JG2020CXCY012) 
当下的分析化学实验教学方法普遍为: 学生课前预习并撰写预习报告, 教师课堂讲解实验原理、 学生操作、教师巡视并纠正学生的不当操作, 学生课后撰写实验报告。尽管这种教学方法在培养学 生的实验技能方面起到了积极作用, 但随着社会的发展以及学生知识背景、学习方式与学习习惯的 变化, 现有的实验教学方式需要完善。具体体现在: (1) 预习效果不理想。预习资料与内容不够丰 富, 预习方式单调, 没有充分利用分析化学在工农业生产中被广泛应用、与日常生活息息相关这一 特点。难以调动学生预习的积极性, 导致学生缺乏对实验的思考, 机械地抄写实验原理与实验步骤。 (2) 课堂教学效果有待提高, 课堂上教师讲得多、学生说的少, 学生的学习主体地位体现得不够充 分, 对学生主观能动性的调动不足, 学生在实验过程中往往不加思考地、机械地按照课本步骤进行 操作, 对于实验每一个步骤的目的和意义理解不足, 在实验过程中出现了问题不能自发解决, 对教 师的依赖程度过重。(3) 实验课后无反馈, 学生学习效果缺乏沟通与交流。现有的分析化学实验教学 环节止步于 “学生撰写报告, 教师批改报告”, 学生对于实验操作中遇到的问题以及所撰写的实验 报告质量如何都是一头雾水。而受限于课程时长的设置, 教师发现学生实验报告的问题, 也很难在 下次实验课中对所有同学的报告进行点评分析。

2018年, 教育部在《关于狠抓新时代全国高等学校本科教育工作会议精神落实的通知》中提出 了建设 “金课” 的战略目标, 要求大学课程应当具有高阶性、创新性和挑战度, 要求教学内容由知 识传授向知识、能力、素质相结合转变, 要求教学形式应具有先进性、互动性, 能培养学生主动探 究的能力。这就要求我们探索更为适当的实验教学方法, 进一步提高教学质量。

案例分析教学法 $(\mathrm{CBL})$ 由哈佛大学首创, 通过设计典型案例再现情境, 引导学生运用理论知识综 合分析, 既加深对理论知识的理解, 又培养学生对理论知识灵活运用的能力 ${ }^{[1,2]}$ 。在课程中融入案例 分析教学, 有利于激发学生的学习兴趣, 建立师生之间的良性互动 ${ }^{[3,4]}$ 。翻转课堂是教师通过合理安 排课堂内外的学习内容, 组织学生进行学习、讨论与讲授的教学方式。翻转课堂以教师对课程内容 的精心设计与教学课堂的恰当组织为核心, 以学生的自主学习和分析讨论为主要组织形式, 改变了 传统课堂 “满堂灌” 的局面, 充分体现出学生在学习过程中的主体地位, 能够有效激发学生的学习 兴趣, 提高教学质量 ${ }^{[5,6]}$ 。混合式教学是当前高校教学改革中普遍采用的方式 ${ }^{[7]}$ 。新冠疫情以来, 微 信、钉钉、腾讯会议等即时通讯软件结合雨课堂以及超星、中国大学慕课等网络资源平台为混合式 教学拓展了新的形式。国内多所高校已利用线上学习平台开展了丰富多彩的混合式教学改革实践, 在 调动学习兴趣、增强师生交流、落实教学反馈、提升教学质量等方面取得了良好效果 ${ }^{[8-10]}$ 。

在本校的分析化学实验教学实践中, 我们将案例分析教学与翻转课堂相结合, 充分发挥案例分 析法理论结合实际的优势, 结合翻转课堂以学生自主学习与分析讨论为主的特点, 调动学生的主观 能动性, 提升学生在学习中的主体地位。通过团队学习的形式, 提升学生的团队协作能力与表达能 力。建立线上交流平台, 实现课程通知、学习资料的发布以及课程学习效果的反馈, 提高学生在课 程预习与撰写实验报告中的表现。此外, 我们还设计了侧重于能力考查的考评方法。通过实践基于 案例分析与翻转课堂的分析化学实验混合式教学, 提升了分析化学实验的教学效果和学习质量。

\section{1 基于案例分析与翻转课堂的混合式教学方法}

\section{1 基于案例分析与翻转课堂的混合式教学方法的设计}

依据建设 “一流课程” 的目标与要求, 我们对实验课程的教学方法进行了重新设计。在新的教 学方法中, 利用互联网线上学习平台, 增加师生互动机会, 突出学生的主观能动性, 注重对能力与 素质的培养。教学方法设计如下。

(1) 教师精选案例并设计分析任务, 学生做好课前预习。教师选择时事新闻、生活现象或其他与 实验课程内容联系紧密的事件作为案例, 依据案例设计3-4个层层递进的问题。教师在课前将设计好 的案例发布在学习通或雨课堂等平台。学生分组, 以案例为主要内容进行自主学习。学生预习应实 现由生活现象到实验现象的理解, 以及由实验现象到化学本质的概括。 
(2) 学生分析讨论案例, 教师纠偏查漏补缺。学生以组为单位(每组2-3名同学)轮流作为主讲进 行案例分析, 其他同学进行提问和补充。教师需认真听取, 并在学生讲完之后对学生准备的内容做 出评价。尤其是纠正学生讲解过程中出现的知识错误、逻辑错漏, 并补充相应的背景知识。翻转课 堂教学中尽量体现学生的课堂主体地位。

(3) 学生操作实验, 教师针对性辅导。学生按照步骤进行实验操作, 教师巡视, 发现学生操作中 出现的问题及时开展针对性辅导。

(4) 学生完成并上传实验报告, 教师评阅并给出反馈意见。线下实验课程结束, 学生整理实验数 据并按照要求完成实验报告, 将报告上传至线上平台。教师评阅实验报告, 指出学生报告中实验步 骤与现象描述、实验数据处理、结果与讨论中的疏漏与谬误, 并将评价意见提交到线上平台, 学生 查阅参考。如实验报告问题较严重, 要求其修改之后重新提交。以上环节可以概括为图1。

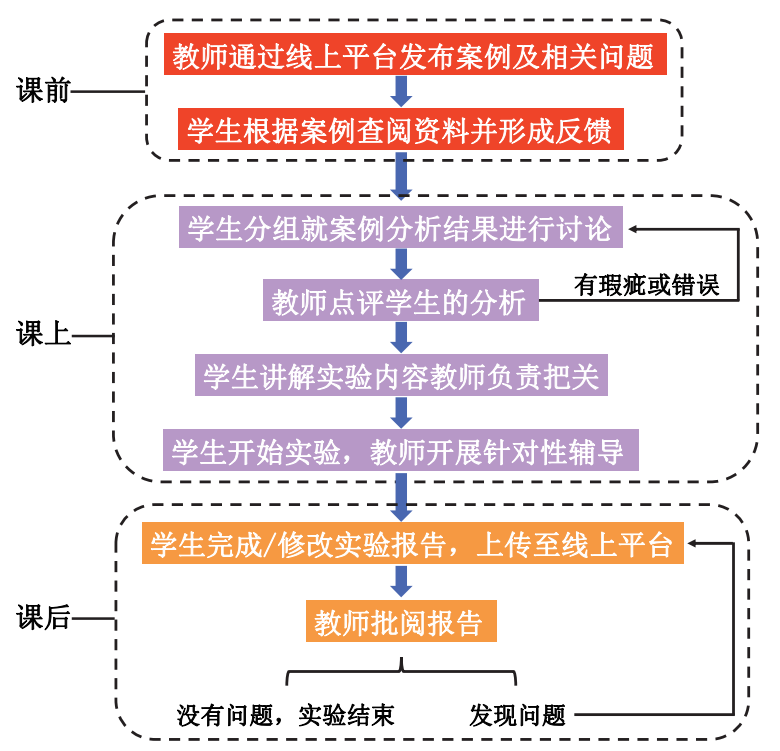

图1 基于案例分析和翻转课堂的分析化学实验混合式教学方法

\section{2 新考评方法的设计}

依据建设 “一流课程” 的要求, 新的教学方式在评价学生表现时更注重学生的主动学习和探究、 专业水平提升、能力与素质的提升等。在评价过程中, 我们确定了 “ 3 种能力 +2 个水平” 的考评目标。 即, 表达能力、逻辑思维能力、团队协作能力, 实验操作水平和整洁水平。各考评目标对应的具体 考评标准及相应赋分值见表1。其中, 团队协作能力的考评由学生互评和教师评价共同组成, 学生根 据承担主要责任及参与团队内部讨论情况互相做出评价, 教师就课堂讨论形式与效果进行评价。

\section{表1 分析化学实验课程的具体考评标准}

\begin{tabular}{clc}
\hline 考评目标 & \multicolumn{1}{c}{ 考评标准 } & 分值 \\
\hline 表达能力 & 课堂分析讨论能做到科学准确(5); 语言流畅(3); 声音洪亮(2) & 10 \\
& 实验报告结构完整(9); 数据处理无误(10); 用语规范(3); 字迹清晰(3) & 25 \\
逻辑思维能力 & 思考及语言表达合乎逻辑、言之成理 & 10 \\
团队协作能力 & 承担团队主要责任(5); 积极参与团队讨论(5); 课堂讨论形式及效果(5) & 15 \\
实验操作水平 & 规范使用滴定管、移液管、容量瓶等仪器(10); 准确判断滴定终点(5); 规范读取、记录实验数据(10); & 30 \\
& 实验操作有条不紊(5) & 10 \\
整洁水平 & 实验中及实验后保持实验台整洁(5); 积极参与值日(5) & 10 \\
\hline
\end{tabular}




\section{2 课堂实践举例}

\section{1 案例的选择与问题的设计}

2018年12月1日，《人民日报》报道了广州警方打掉一个生产销售 “毒鸡脚”的黑作坊的新闻。 黑作坊用双氧水对变质的原材料进行漂白处理, 制作出的鸡爪具有食品安全隐患。最终, 黑作坊被 查封, “毒鸡爪”被销毁。

1) 你知道视频中的毒鸡爪为什么要用过氧化氢处理吗?

2) 如果你是技术检测人员, 你会怎么设计方案测定过量的过氧化氢? 测定原理是什么?

3) 如果食品中存在其他能够让高锰酸钾褪色的物质, 该如何解决这一问题?

4) 国家标准方法是如何测定食品中过量的过氧化氢的? 实验课本上的方法与国家标准方法有 什么区别?

\section{2 学生预习}

在实验课程开始前一周, 将案例新闻视频及以上 4 个问题发布至线上平台。由学生自由分组进行 预习。在预习过程中, 每组同学需要围绕 4 个问题完成相关资料搜集、共同讨论分析、提出解决方案、 制作课件等任务。

\section{3 翻转课堂及相关考评}

在课堂上, 首先由学生进行案例分析, 教师在此过程中观察、记录所有学生的表现, 就学生讨 论分析时的语言表达、逻辑思维、团队协作进行考评。

通过查阅资料, 学生都能了解双氧水具有较强的氧化性, 能将变质鸡爪中的色素氧化清除从而 实现漂白, 这是黑作坊使用过氧化氢处理 “毒鸡爪” 的原因。因此, 检测过量的过氧化氢, 能帮助 判断食品是否安全。学生共找到 3 种检测过氧化氢的方法。第一种是高锰酸钾法。利用高锰酸钾在稀 硫酸溶液中与过氧化氢发生定量氧化还原反应的原理测定过氧化氢。第二种方法是碘量法。食品中 的强氧化剂在稀硫酸中使碘化钾氧化, 产生定量的碘, 生成的碘以淀粉作指示剂, 用硫代硫酸钠标 准溶液滴定间接测出强氧化剂含量。加入过氧化氢酶分解去除试样中的过氧化氢, 用硫代硫酸钠标 准溶液滴定去除过氧化氢后的其他氧化剂含量。两次滴定结果之差可计算得到样品中过氧化氢的含 量。第三种方法是钛盐比色法。过氧化氢在酸性溶液中与钛离子生成稳定的橙色络合物, 络合物在 $430 \mathrm{~nm}$ 处的吸光度与过氧化氢含量成正比, 用比色法测定样品中过氧化氢的含量。通过对三种方法 检测原理的分析, 得出结论: 碘量法和钛盐比色法具有更好的选择性和抗干扰能力, 而高锰酸钾法 则具有操作简单的优势。

接下来, 将学生分成 $A 、 B$ 两大组。A 组同学用三种方法分析市售双氧水中过氧化氢的含量, B组 同学用三种方法分析市售泡椒风爪样品(由实验人员提前准备)中过氧化氢的含量。在实验过程中, 教 师及时纠正学生的错误实验操作, 并在学生需要的时候提供必要指导, 完成对学生实验操作水平的 评价。

实验结束后, 教师组织学生对实验结果进行讨论分析。组内同学讨论分析不同方法分析相同样 品的结果, 组间同学对比相同方法分析不同样品的实验现象。最终得出结论: 在分析市售双氧水中 过氧化氢含量时, 三种方法的结果不存在显著性差异; 而在分析市售泡椒凤爪样品时, 高锰酸钾法 因为选择性不佳产生较大正误差, 碘量法与钛盐法的结果不存在显著性差异。由此, 引导学生认识 到分析方法的适用范围以及选择性问题。同时, 也让学生切实认识到分析化学是一门具有广泛应用 价值的学科, 课堂中学到的知识具有很强的实用性, 以此增加学生对分析化学实验课程的兴趣以及 对学科学习的热爱。最后, 检查学生实验台的整洁与值日情况, 做出评分。

课程结束后, 学生完成实验报告并上传至学习通平台。教师线上评阅, 并将报告中出现的问题 进行标注和说明。学生查阅教师批阅后的报告, 可对教师指出的问题进行一次修正。教师对学生的 实验报告进行评分。 


\section{3 对新教学方法的反思}

在上一个分析化学实验教学周期内, 我们在酸碱滴定、配位滴定、氧化还原滴定法等 3 个专题共 安排4次案例分析与翻转课堂结合的教学方法实践。教学效果明显优于未进行新方法的教学课堂。主 要体现在: (1) 学生预习有的放矢, 参与学习的主动性更强, 预习效果显著提升; (2) 学生在课堂上 积极发言讨论, 师生互动、生生互动增多, 学生的课堂主体地位充分展现; (3) 团队协作, 分工查找、 整合资料, 分工配制溶液提高实验效率等, 提升了学生的团队协作能力; (4) 学生敢于突破自我, 选 择更具有挑战性的课堂展示方式, 例如有一组同学在讲解锌铋混合溶液连续滴定时全程使用英语口 述和英文板书, 让人印象深刻; (5) 学习效果有反馈, 学生能够知晓实验报告中存在的问题, 在后续 的实验报告撰写中尽量避免, 实验报告的质量有较大提升。在课后的调查中, 多数同学表示这一教 学方法能让自己有更多收获, 期待未来的课程中能够使用类似的教学方法。

然而, 这一教学方法也存在不足。比如, 有部分同学反映需要在课前查阅大量资料, 无形之中 增加了更多学习任务和压力。在未来的教学实践中, 这可能需要教师凝练案例设计并分解预习任务, 帮助学生提高效率。另外, 这一教学方法中需要对学生进行评价的项目太多, 在只有一名教师授课 的情况下, 可能出现照顾不到所有学生的情况。在未来的教学实践中, 可以尝试通过增加课堂教学 环节教师数量的方法来解决。

\section{4 结语}

本文设计了基于案例分析与翻转课堂的混合式教学方法, 在分析化学实验教学中进行了实践。 通过一个教学周期的观察与比较, 这一教学方法能够解决传统实验教学方法的不足, 帮助提高学生 学习积极性, 帮助学生提升表达能力和实验操作水平, 还能够激发学生挑战高难度学习任务的热情, 具有一定的推广借鉴价值。借助与新教学方法对应的考评方法, 实现了对学生能力表现的考评。目 前这一教学方法还存在学生任务过多以及课堂教学中教师不足的问题, 在未来的教学实践中可通过 分解学习任务、增加教师数量的方式解决。我们相信, 在进一步完善现有问题的基础之上, 这一教 学方式能够更好地助力 “一流课程”建设。

\section{参 考 文 献}

[1] Barnes, L. B.; Christensen, C. R.; Hansen, A. J. Teaching and the Case Method: Texts, Cases, and Readings, 3rd ed.; Harvard Business School Press: Boston, MA, USA, 1994; pp. 89-100.

[2] 杨蓉, 钟世华, 肖小明. 化学教育(中英文), 2018, 39 (16), 57.

[3] 杜振霞, 杨屹, 苏萍, 吕超, 胡高飞, 张丽娟. 化学教育(中英文), 2017, 38 (20), 14.

[4] 何婧琳, 曹忠, 李丹, 张玲, 谭淑珍, 陈平, 李伟. 大学化学, 2016, 31 (8), 27.

[5] 刘盈, 张镖, 王丽丽, 姚小丽, 张素中. 化学教育(中英文), 2020, 41 (20), 92.

[6] 张伟, 王林涛, 戚小强, 林海燕, 陈芳,袁栋. 生命的化学, 2021, 41 (7), 1403.

[7] 陈算荣. 高教学刊, 2016, 31 (7), 15.

[8] 杨天方. 高教学刊, 2020, $156(34), 93$.

[9] 龙旭, 唐志书, 唐于平, 史亚军, 靳如意, 郭惠, 孟庆华, 李佳佳, 张拴, 李小蓉. 化学教育(中英文), 2020, 41 (20), 86.

[10] 邵伟, 张万群, 胡万群, 李维维, 盛翔, 柯玉萍, 杨凯平, 孙晴, 朱平平. 大学化学, 2021, 36 (7), 2012012. 